\title{
HIGH EFFICIENCY CIRCUITAL SCHEME OF HARD RADIATION ON BASE OF COMPACT ELECTRON ACCELERATORS
}

\author{
V.Grishin", S.Likhachev \\ Nuclear Physics Institute of Moscow State University, 119899 Moscow, Russia
}

\begin{abstract}
The new physical schemes of X-ray and $\gamma$ - radiation sources are considered in which the process of photon emission happens at multiple cross by circulating electrons of the thin target The sources of a bremsstrahlung radiation with additional acceleration of radiating electrons and monochromatic radiation with usage of the crystalline target are discussed. The computer model operation confirms high efficiency of schemes, which allow essentially to increase an yield of both hard and monochromatic photons at maintenance of compact device sizes that is specially important at development of different sources.
\end{abstract}

\section{INRIDUCTION}

Electron sources of hard X-ray and $\gamma$-radiation (HRS) discover broad applications in diverse examinations, and it is extremely important to raise their efficiency which is not exceeding 5-10\% [1,2]. Therefore actively there are searches of more effective devices HRS, see for example [2-4]. So, in Ref. [2] a series of the schemes are surveyed in which electrons, circulating in a special magnetic field, repeatedly intercross thin target. This operation mode allows essentially, approximately three times to increase overall radiation yield. Two new perspective schemes applying further development of a circuital principle are considered below.

\section{HARD RADIATION SOURCE}

One of most promising is a scheme with the prolonged cycle of radiation on a thin target. In the traditional schemes due to presence of various absorbing processes, the radiation occurs on the average at electron energy smaller than initial one. Opposite in a mode with multiple crossing of a thin target and simultaneous energy compensation, the radiation occurs on the average at electron energy close to initial one. Therefore photon share with the maximal energy should here appreciably grow.

Possible scheme of such source is an electronic magnetic field (can be utilized permanent magnets) and induction on an interior orbit core passed through poles

*grishin@depni.npi.msu.su

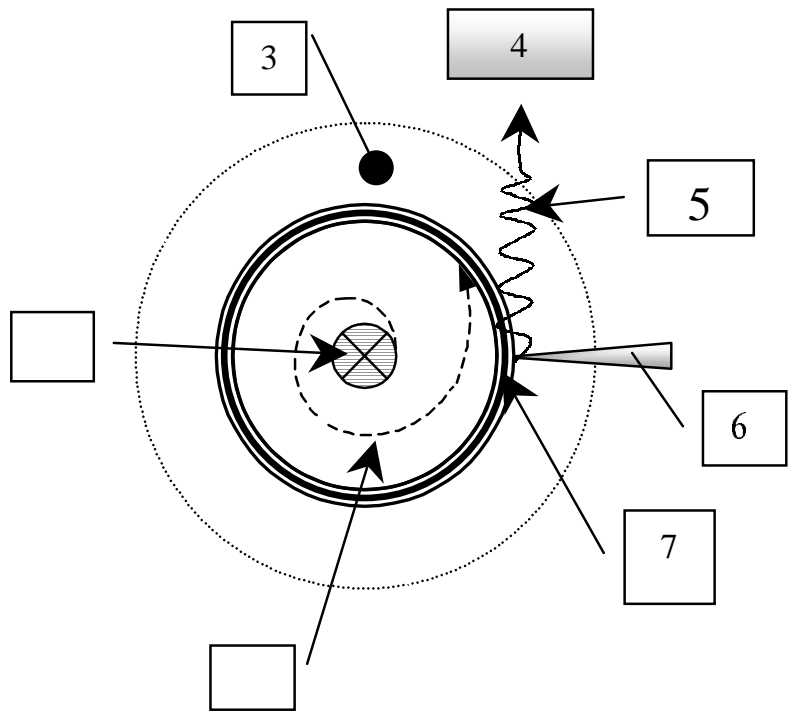

Fig. 1. Recirculator - source of a hard radiation. 1 Trajectory of electrons at an initial acceleration (schematically). 2 - Induction heart. 2 - Magnetic pole. 4 -Radiation detector. 5 - Gamma radiation. 6 - Wedge target. 7 - Trajectory of electrons near to an orbit of radiation.

cyclotron with an induction beam acceleration, Fig. 1 . The system consists of flat magnetic poles creating a constant

stimulating a accelerating field. The duty cycle starts with injection of electrons. Further being accelerated, the electrons displace up to some exterior trajectory. Directly to last the special wedge target is flanked. On reaching by electrons this orbit, the accelerating cycle is been prolonged. Then the electrons will displace out crossing the target, losing energy and radiating photons. The width of the target grows with orbit radius. Due to this a dynamic balance between power loss of a particle and its acceleration is automatically erected on some radius, and this balance will be supported up to the end of an accelerating cycle. Then the electrons are dropped on an additional target, radiating the last portion of photons, and the cycle is iterated.

The dynamic stability of electrons at acceleration down to orbits of radiation is ensured due to a natural wane of 
quantity of a magnetic field on a rim of poles . By the data [5], the stable circulations should be watched though in an emissive mode the electrons experience a dispersion in targets deflecting their trajectories. According to the prognoses [2] the similar scheme has high efficiency, and the spectrum of radiated photons is enriched by both weak and hard photons.

These prognoses were tested by computer modelling applying the software package GEANT [6], specially adapted for motion study of particles in different electromagnetic fields [5]. The model operation is restricted by the analysis of the most relevant stage of radiation when the particles with energy $\mathrm{E}_{0}$ are dropped on the wedge target placed on corresponding orbit in axial - symmetric, feebly waning at removal from centre a magnetic field . Rotating and radiating electrons obtain an additional portion of energy $\mathrm{dE}$ after very target crossing. The prolonged cycle of radiation is stopped on reaching a definite stage.

Modeling confirmed a dynamic stability of electrons, the trajectories which did not fall outside the limits of a current tube, in spite of the fact that "jumps"of orbits on each turnover are noticeable since a dynamic balance is fulfilled only on the average (last circumstance reflects casual character of dispersion and radiation processes ; the program imitated this fact by a Monte-Carlo method). The amount of traced circulations reaches up to hundreds turnovers (extinction of a prolonged cycle for separate particles is caused by code limits). Therefore energy $\mathrm{dE}$ is picked enough major down to tenth $\mathrm{MeV}$ for a turnover.

Let's cite some data of one of modeling variants with parameters: initial energy of electrons $\mathrm{E}_{0}=20 \mathrm{MeV}, \mathrm{dE}$ $=0.1 \mathrm{MeV} /$ turnovers. A mean value of aggregate energy reaches $29 \mathrm{MeV}$ at 300 revolutions though the separate particles were traced up to thousand turnovers. The results of modeling are represented on four spectra (obtained at equal particle amount of 5000) illustrated in fig. 2 and 3 and allowed to judge efficiency of the proposed scheme.

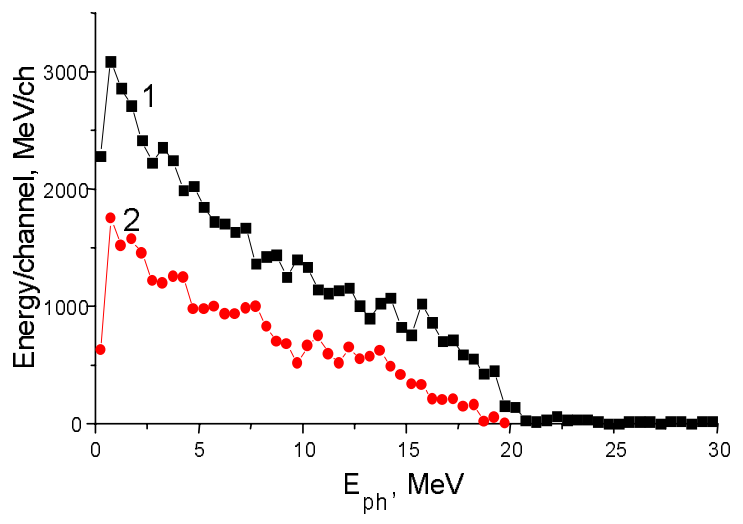

Fig.. 2 of Spectrum of bremsstrahlung radiation of electrons with initial energy $20 \mathrm{MeV} .1$ - Energy intensity of radiation in circulating mode reduced by factor 20/29. 2 - Energy intensity of radiation in traditional mode.

Fig. 3 allows to judge, what advantages there is a tendered mode as contrasted to conventional one in a case when the particles gain en total equal energies .

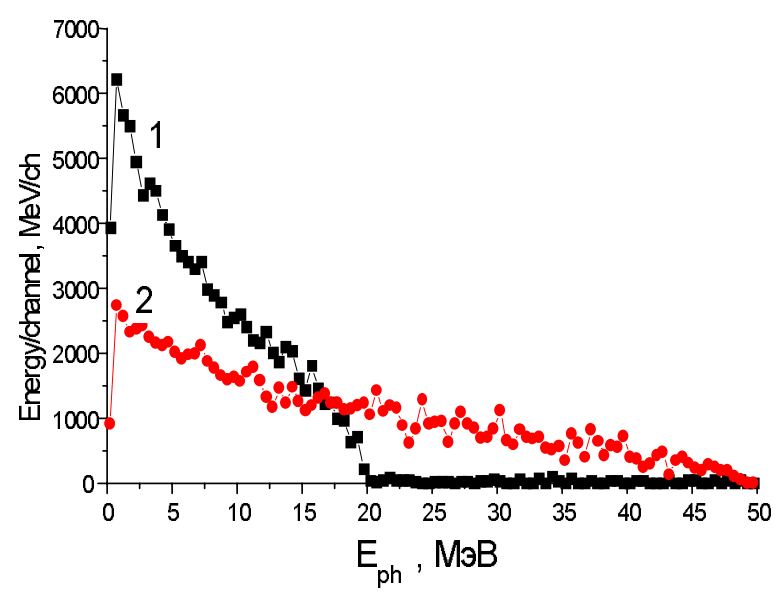

Fig. 3. Radiation spectra. 1 - Energy intensity of photons radiated in circulating mode and in traditional one with electron energy at $20 \mathrm{MeV}$ at finale. 2 - Energy intensity of photons radiated in traditional mode with electron energy at $50 \mathrm{MeV}$.

This spectra visually reflect peculiarities of circuital and traditional radiation, demonstrating advantages first on quality of a spectra and on total energetics of radiation. So the total energy of photons in circulating mode (only !) two times surpasses the same quantity in traditional scheme. The data of fig. 3 indicates that for deriving by a conventional mode a stream of $\gamma$-quantums similar circuital one at energy close $20 \mathrm{MeV}$, it is necessary to utilize an accelerator in 2.5 times greater on the sizes.

\section{MONOCHROMATIC SOURCE}

Source of tunable quasi-monochromatic X-ray and $\gamma$ photons can be designed on base of parametric X-rays radiation (PXR) of relativistic electrons in crystals $[7,8]$. However essential deficiency of this project is its small intensity. In many respects it is bound with necessity to utilize thin targets in practice. Really, the more thick target produces both an angular dispersion of electrons and strong absorption of emitted photons in the target matter.

Nevertheless efficiency of a such source can be sharply increased if generating electrons will multiply intercross thin crystalline target. The experimental scheme is represented in a fig. 4. 


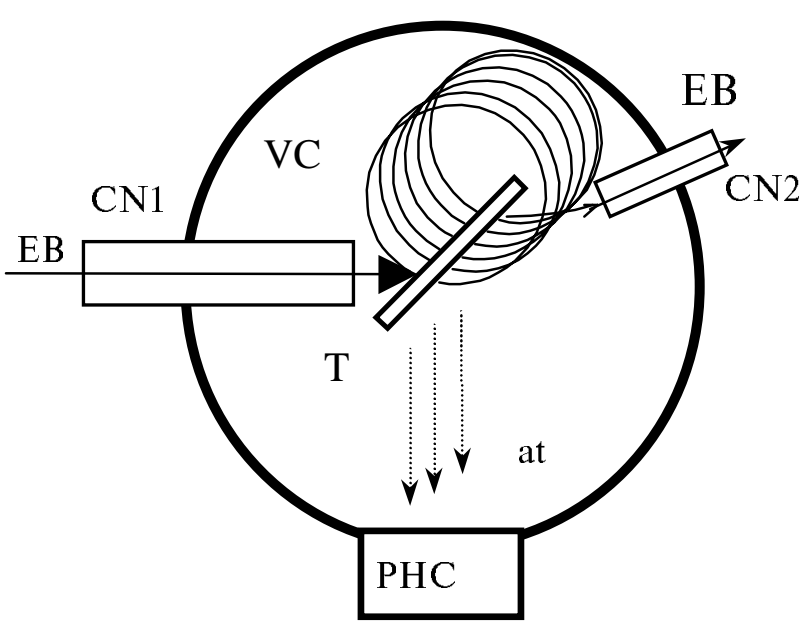

Fig. 4. The scheme of a source of monochromatic radiation. $\mathrm{VC}$ is a vacuum chamber, $\mathrm{M}$ crystalline target, EB is beam coupling, PHC is channel of an output at photons, $\mathrm{CN} 1$ and $\mathrm{CN} 2$ are in- and output channels.

Electron beam is injected through the special channel in a vacuum chamber with the crystalline target. The electrons circulate in a magnetic field, repeatedly crossing the target, generating PXR and simultaneously displace along the target. Then they are output through the special channel. PXR generated in crystal under Bragg's angle $\theta_{\mathrm{B}}$, leaves through the photon channel.

. The required configuration of a magnetic field can be obtained using magnetic poles of a rather simple form: two pole-parallelepipeds symmetrically are disposed above and under the target on all to its length. Poles are slightly shifted across the target plane. In this case dispersion of a field on edges of poles ensures a focusing of circulating particles. Due to pole displacement, a lateral drift of particles is created. Note here magnetic focusing must return the electrons on target In parallel to initial trajectory.

For testing of scheme a method of computer modeling also is utilized. The design is oriented on usage of an electron accelerator with energy 2-5 MeV. Bragg's angle is $45^{\circ}$. The target is the crystalline silicon plate which is cut so, that the vector of an inverse lattice $\langle 111\rangle$ lies along its surface. The target thickness is equal 20-30 microns. This scheme allows to generate polarized (tunable by variation of Bragg's angle ) radiation with photon energies from several up to ten $\mathrm{keV}$.

Spectrum is defined in the basic by angular size $\Delta \theta$ of the photon channel. So the relative width $\Delta \omega / \omega$ of line radiation is about $\Delta \theta / \tan \theta_{\mathrm{B}}$.

According to Ref. [9] an average current for silicon crystal hitting makes about $0,5 \mathrm{~A}$ at bunch radius in 1 $\mathrm{mm}$. There are also restrictions bound with problems of space charge of circulating particles. In continuous accelerators with a current can be up to several milliamperes and radiation intensity up to some tens W/St/A.

Results of modeling shows that the particles can make tens turnovers, and not less than 10-20 circulations are made in limits of a demanded radiation angle. Then the effective length of multiple electron radiation sharply will increase. Therefore source tested can be comparable on capacity with devices using much higher electron energy

Thus, as well as in a case HRS with the prolonged cycle of radiation, the analysis displays an opportunity of making of an effective monochromatic radiation source. Moderate consumed power and compact sizes of device (compare with Ref. [10]) unclose actual opportunities for the broad and diversiform applications.

\section{REFERENCES}

[1] Findlay D.J.S.//Nucl.Instr. and Meth. in Phys. Res. 1990. B50.P.314.

[2] Grishin V.K., Ishkhanov B.S.,Shvedunov V.I.// Vestnic Moskovskogo Universiteta. Ser.3. 1996. N1. P.83.

[3] B.Bogdanovich, V.Kudinov, A.Nesterovich, Yu.Pomasan, E.Tsygankov, V.Janenko, Proceeding of "PAC'97"(Vancouver, 1997), 1998 IEEE, P.276

[4] V.V.Kaplin, L.W.Lombardo, A.A.Mihalchuk et al.//Nucl.Instr.and Meth. in Phys.Res.1998. B 145.P.244.

[5] V.Grishin, B.Ishkhanov, S.Likhachev, Proc.of Int . Conf. PAC97, APS-IEEE, 1997 P.3866.

[6] Brun R., Bruyant., Maire M. et al. GEANT3 (User manual). GERN,Geneva, Switzland, 1990.

[7] M. Ter-Mikaelian. High energy electromagnetic processes in condensed media (Wiley-Interscience. NewYork, 1972 ).

[8] N.Nasonov, A.Safronov. Proc.Int.Symp. RREPS-93, Oct. 1993, Tomsk, Russia.

[9] R.Fiorito, Rule D.W., Piestrup M.A. et al.//NIM B 79,758-761 (1993).

[10] A.Andriyashkin, V. Kaplin, M.Pristrup et al.// Appl. Phys. Lett., v. 72, N 11, p.1385 (1988). 